\title{
Small-signal modulation and temperature dependence of the tunneling injection laser
}

\author{
L. Davis, H. C. Sun, H. Yoon, and P. K. Bhattacharya \\ Solid State Electronics Laboratory, Department of Electrical Engineering and Computer Science, \\ The University of Michigan, Ann Arbor, Michigan 48109-2122
}

(Received 4 January 1994; accepted for publication 30 March 1994)

\begin{abstract}
Recently, we demonstrated a novel laser structure, called the tunneling injection laser, where the electrons are injected into the active lasing quantum well region via tunneling. High performance results for this device have now been demonstrated. $A T_{o}$ of $160 \mathrm{~K}$ was found from lemperature-dependent measurements $\left(25-70^{\circ} \mathrm{C}\right)$. High differential gain $\left(5.5 \times 10^{-16} \mathrm{~cm}^{2}\right) \mathrm{and}^{-}$ modulation bandwidth $(12.5 \mathrm{GHz})$ have been attained relative to other single quantum well lasers.
\end{abstract}

The problem of carrier injection into the well region of a quantum well laser, including the transport across the separate confinement heterostructure ( $\mathrm{SCH}$ ) region and the carrier relaxation from the three-dimensional to twodimensional region, has been the subject of much recent interest. ${ }^{1,2}$ Several techniques have been used to overcome some of the limitations imposed by an SCH-type laser, however the fundamental limitations still remain. In an SCH laser, the electron-hole $(e-h)$ stimulated emission time at high injection approaches the relaxation time from the barriers into the quantum wells. As a result, the carrier distribution in the quantum well becomes "hot" and is not described by a quasi-Fermi distribution. This can create serious limitations for the laser performance by introducing gain-compression enhanced Auger rates and other hot-carrier related effects.

Recently, we demonstrated a novel quantum well laser structure, ${ }^{3}$ called the tunneling injection laser (TIL), in order to overcome the carrier relaxation-induced limitations of SCH lasers. The electrons are injected into the active lasing region via tunneling. One expects tremendous advantages from such a structure. The carrier distribution in the lasing well of the TIL is governed by the thermalization time of carriers injected by tunneling $\left(\sim 1 \mathrm{ps}^{4}\right)$, rather than by the "phonon bottleneck" of carriers injected over the barriers. This will lead to a carrier distribution in the active well that is described by a quasi-Fermi distribution even at high injection levels. This will improve the gain at a selected energy and dramatically reduce hol carrier effects like Auger recombination. High performance results for this device have been realized in the latest structures, revealing some of its ultimate potential. The temperature-dependent characteristics have been investigated, as well as the small signal modulation performance.

Since the theoretical optimization was being conducted concurrently with the device fabrication, it was impossible to know a priori what the optimal tunneling structure would be. Structures were grown with different GaAs injection layer thicknesses (700-1200 $\AA$ ), different indium compositions in the active well $\left(\mathrm{In}_{0.10} \mathrm{Ga}_{0.90}\right.$ As and $\mathrm{In}_{0.20} \mathrm{Ga}_{0.80} \mathrm{As}$ ), and different widths of the resonant tunneling well (40 and $45 \AA$ ). For the structures that we have fabricated, the best results were obtained from the sample in Fig. 1(a), grown by molecular beam epitaxy. It should be noted that this may not be the optimal structure, but rather the device with the best results so far. The AlAs barriers ensure that the electron injection will be by tunneling, since the AlAs provides a barrier of more than $400 \mathrm{meV}$ to thermionic emission in the conduction band. The width of the AlGaAs inner cladding was chosen to maximize the optical overlap with the lasing well. The energy band diagram and the calculated optical mode profile of the device are shown in Fig. 1(b).

Single mode ridge lasers were made by standard photo-

\begin{tabular}{|c|c|c|}
\hline $0.1 \mu \mathrm{m}$ & CONTACT GaAs & $\mathrm{p}^{+}\left(5 \times 10^{18} \mathrm{~cm}^{-3}\right)$ \\
\hline $1.0 / / \mathrm{m}$ & OUTF,R CLAD $\mathrm{Al}_{0.60} \mathrm{Ga}_{0.40} \mathrm{As}$ & $\mathrm{p}\left(5 \times 10^{-17} \mathrm{~cm}^{-3}\right)$ \\
\hline $1000 \AA$ & INNER CLAD $\mathrm{Al}_{0.30} \mathrm{Ga}_{0.70} \mathrm{As}$ & $\mathfrak{i}$ \\
\hline $80 \AA$ & ACTIVE WELL In ${ }_{0.20} \mathrm{Ga}_{0.80} \mathrm{As}$ & $\mathrm{i}$ \\
\hline $20 \AA$ & Resonant Tunneling Barrier AlAs & $i$ \\
\hline $40 \AA$ & Tunneling Well $\operatorname{In}_{0.20} \mathrm{Ga}_{0.80} \mathrm{As}$ & $i$ \\
\hline $20 \AA$ & Tunneling Barrier AlAs & $\mathrm{i}$ \\
\hline $1000 \AA$ & INNER CLAD GaAs & i \\
\hline $1.0 \mu \mathrm{m}$ & OUTER CLAD Al $\mathrm{L}_{0.80} \mathrm{Ga}_{0.40 \mathrm{As}}$ & $\mathrm{n}\left(5 \times 10^{17} \mathrm{~cm}^{-3}\right)$ \\
\hline \multirow[t]{2}{*}{$0.7 \mu \mathrm{m}$} & CONTACT GaAs & $\mathrm{n}^{4}\left(5 \times 10^{18} \mathrm{~cm}^{-3}\right)$ \\
\hline & S.I. GaAs (100) Substrate & \\
\hline
\end{tabular}

(a)

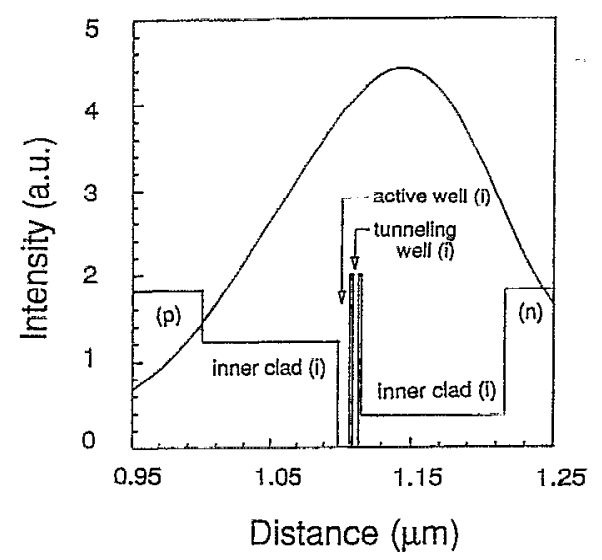

(b)

FIG. 1. (a) GaAs-based tunneling injection laser with a $\operatorname{In}_{0.2} \mathrm{Ga}_{0.8}$ As lasing well grown by molecular beam epitaxy; and (b) the energy band diagram and the calculated optical mode profile of the device. 


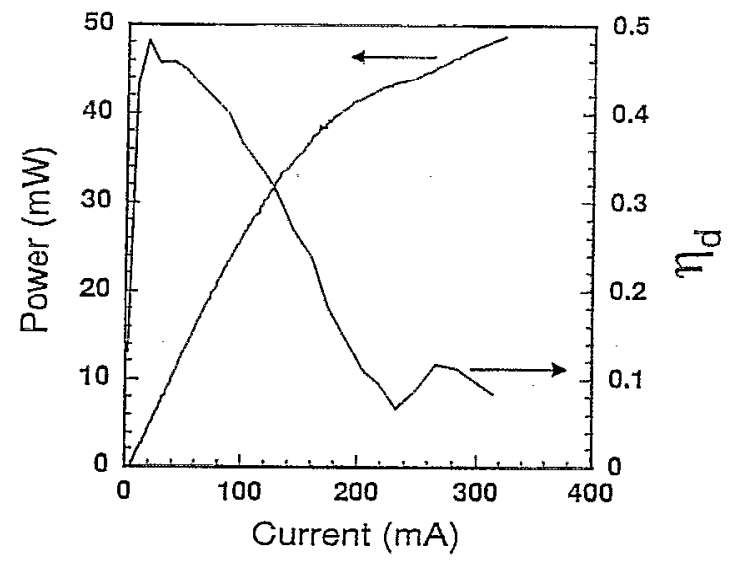

FIG. 2. A typical $L-I$ and differential efficiency plot for a single mode tunneling injection laser with $L=200 \mu \mathrm{m}$ at $T=300 \mathrm{~K}$.

lithography and wet and dry etching. Many devices of different lengths were tested in order to form a good overall picture of the device operation. $L-I$ plots for 20 of the devices with $\mathrm{In}_{0.20} \mathrm{Ga}_{0.80}$ As active regions were taken as a function of temperature $\left(25,40,55\right.$, and $\left.70^{\circ} \mathrm{C}\right)$. All measurements are pulse biased, with $0.4 \mu$ s pulses and a 1:500 duty cycle. A typical $L-I$ characteristic is shown in Fig. 2, with the associated differential efficiency. The rapid roll off of the differential efficiency is attributed to heating, since the device is treated $p$-side up and is not indium mounted to the heat sink. This device shows an initial slope efficiency, $d P /$ $d I$, of $0.31 \mathrm{~mW} / \mathrm{mA}$. A plot of $1 / \eta_{d}$ versus cavity length for 15 devices yields an internal quantum efficiency $\eta_{i}=0.56$ and a guide loss of $\alpha_{\mathrm{i}}=13 \mathrm{~cm}^{-1}$ at room temperature. These are still not optimal, showing further development needs to be done. The lowest room temperature $I_{\mathrm{th}} \sim 3 \mathrm{~mA}$ for the best $200 \mu \mathrm{m}$ device. Fitting the threshold current variation with temperature to the phenomenological expression, $I_{\text {th }}$ $=I_{o} e^{T / T_{o}}$, the value of $T_{o}$ can be extracted from 25 to $70^{\circ} \mathrm{C}$. The best $T_{o}$ for the tunneling injection laser is significantly better than the best $T_{o}$ for the $\mathrm{SCH}$ multiquantum well (MQW) lasers made from our molecular beam epitaxy (MBE)-grown material, ${ }^{5}$ as seen in Fig. 3. This shows the potential that this structure has for producing high performance devices.

The measured threshold current density versus cavity length is used to determine the transparency current density and the gain coefficient using the logarithmic gain approximation. ${ }^{6}$ By performing a linear fit of a plot of $\alpha_{i}+(1 / 2 L) \ln \left(1 / R_{1} R_{2}\right)$ vs $\ln \left(\eta_{i} J_{\mathrm{th}}\right)$, the transparency current density and the gain coefficient can be extracted. The transparency current density does not increase much with temperature- $J_{\mathrm{tr}}=263 \mathrm{~A} / \mathrm{cm}^{2}$ at $25^{\circ} \mathrm{C}$ and $309 \mathrm{~A} / \mathrm{cm}^{2}$ at $70^{\circ} \mathrm{C}$-this indicates that the basic design is good. The transparency current density is a function of the materials chosen (bandstructure, optical confinement factor, etc.), and, as such, is a good figure of merit when comparing device designs. For our structure the transparency current is too high, and future designs will have to address this. For the same layer structure shown in Fig. 1(a) except with a $\mathrm{In}_{0.10} \mathrm{Ga}_{0.90} \mathrm{As}$ active region, the transparency current was

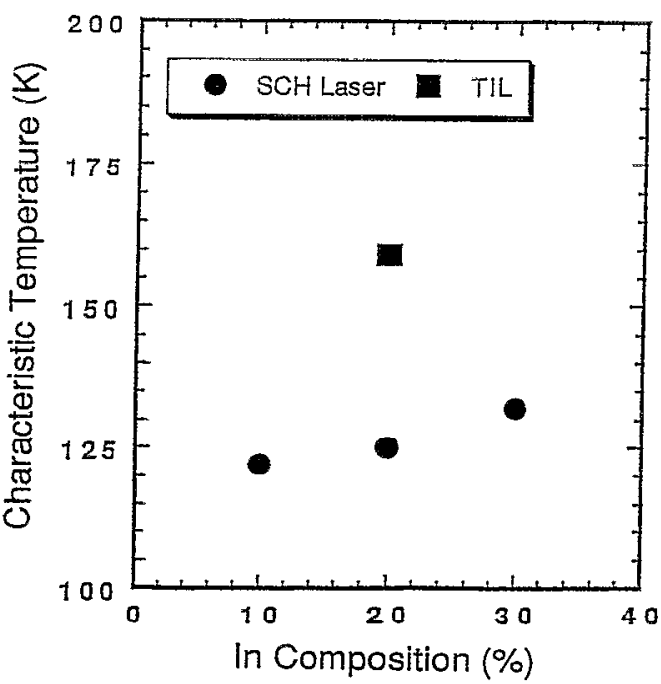

FIG. 3. A plot of $T_{o}$ vs indium composition, showing the superior performance of the tunneling injection laser against standard SCH-MQW lasers.

measured to be $80 \mathrm{~A} / \mathrm{cm}^{23}$. The lower $J_{\text {tr }}$ for the devices with less indium in the active region is contrary to what typically occurs for standard SCH-MQW devices. In the tunneling injection laser, the increased $J_{\mathrm{tr}}$ for increased indium in the active region may be due to the increased potential energy that must be lost by the nonresonantly injected electrons before reaching the lasing energy level. This may require changes in the well width and indium composition of future designs.

Once the transparency current density and the cavity losses are known, one can extract the differential gain, $g^{\prime}$, using $^{7}$

$$
J_{\mathrm{th}}=J_{\mathrm{tr}}+\frac{q d}{\eta_{i} \tau_{e} \Gamma g^{\prime}}\left(\alpha_{i}+\frac{1}{L} \ln \frac{1}{R}\right),
$$

where $J_{\text {th }}$ is the threshold current density, $J_{\text {tr }}$ is the transparency current density, $d$ is the active layer thickness, $\alpha_{i}$ is the cavity loss, $L$ is the cavity length, $R$ is the facet reflectivity, and $\tau_{e}$ is the carrier lifetime at threshold. The variation of the differential gain with temperature is shown in Fig. 4. The values of differential gain shown here are very good when compared to any semiconductor laser, and represent the high-

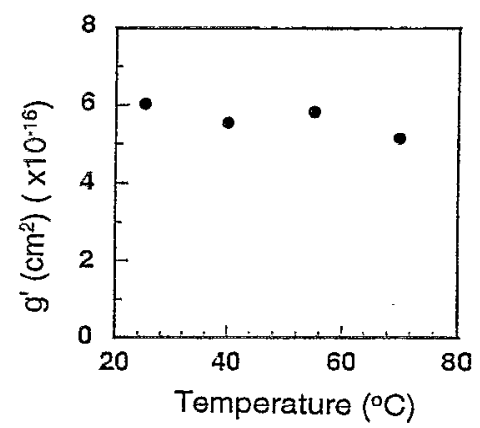

FIG. 4. The differential gain variation with temperature, showing record high differential gain values for a single quantum well laser. 


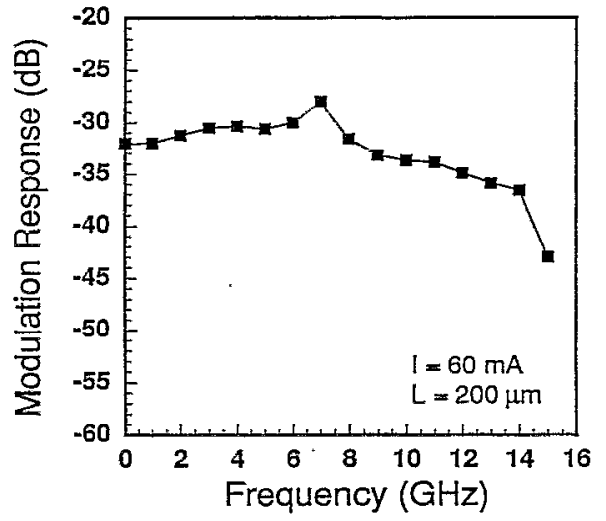

FIG. 5. The best modulation performance of the tunneling injection lascr, showing the highest $3 \mathrm{~dB}$ modulation bandwidth for a single quantum well device.

est differential gain for any single quantum laser device. ${ }^{8}$ The theoretically predicted value for differential gain $\left(\sim 2 \times 10^{-14} \mathrm{~cm}^{2}\right)^{9}$ is still about $33 \times$ larger than the experimentally demonstrated values $\left(6 \times 10^{-16} \mathrm{~cm}^{2}\right)$, showing that considerable experimental work still needs to be done. In comparison to other work, the best differential gain reported for a single quantum well laser is $3.0 \times 10^{-16} \mathrm{~cm}^{2}$ and for a MQW laser is $5.0 \times 10^{-15} \mathrm{~cm}^{2,8}$ furthermore, these devices had $35 \%$ indium in the active region, while the tunneling injection lasers tested here has only $20 \%$. Such a large differential gain should lead to a reduced linewidth enhancement factor and chirp, ${ }^{10}$ which we are presently investigating.

The high differential gain for these tunneling injection devices is paralleled in the tremendous modulation response of this laser. This is expected since the resonance frequency is proportional to the square root of the differential gain. The best $3 \mathrm{~dB}$ modulation data for a $200-\mu \mathrm{m}$-long device is shown in Fig. 5, showing a $12.5 \mathrm{GHz}$ modulation response at a low drive current of $60 \mathrm{~mA}$. This is the highest $3 \mathrm{~dB}$ modulation bandwidth for a single quantum well device. ${ }^{12-14}$
Unfortunately, further increases in the drive current did not lead to increases in the modulation response, as one would expect. This is attributed to changing power distribution in the modes, and current leakage in the ridge waveguide devices. Nevertheless, the modulation performance is excellent, and further record results can be expected from further device optimization.

In conclusion, we have described high performance results for the tunneling injection laser. A $T_{o}$ of $160 \mathrm{~K}$ was found, higher than our standard SCH-MOW laser structures. These single quantum well lasers show a record-high differential gain $\left(5.5 \times 10^{-16} \mathrm{~cm}^{2}\right)$ and modulation bandwidth (12.5 $\mathrm{GHz}$ ), and show tremendous potential for further improvement.

The work is being supported by the Office of Naval Research under Grant N00014-90-J-1831 and the Ballistic Missiles Development Organization (Army Research Office Grant DAAH04-93-G-0034).

${ }^{1}$ W. Rideout, W. Sharfin, E. Koteles, M. Vassell, and B. Elman, Photon. Tech. Lett. 3, 784 (1991).

${ }^{2}$ R. Nagarajan, M. Ishikawa, T. Fukushima, R. S. Geels, and J. E. Bowers, J. Quantum Electron. 28, 1990 (1992).

${ }^{3}$ H. Sun, L. Davis, S. Sethi, J. Singh, and P. Bhattacharya, Photon. Tech. Lett. 5, 870 (1993).

${ }^{4}$ Y. Lam, Ph.D. thesis, University of Michigan, 1993.

${ }^{5}$ H. C. Sun, Ph.D. thesis, University of Michigan, 1993.

${ }^{6}$ P. Mcllroy, A. Kurobe, and Y. Uematsu, J. Quantum Electron. 21, 1958 (1985).

${ }^{7} \mathrm{P}$. Bhattacharya, Semiconductor Optoelectronic Devices (Prentice Hall, Englewood Cliffs, NJ, 1993), p. 273.

${ }^{8}$ S. Offsey, W. Schaff, L. Lester, L. Eastman, and S. McKernan, J. Quantum Electron. 27, 1455 (1991).

${ }^{9}$ P. Bhattacharya, L. Davis, H. C. Sun, H. Yoon, S. Sethi, J. Shuttlewood, I. Vurgaftman, and J. Singh, presented at SPIE's International Symposium and Conferences on Laser Diode Technology and Applications, Los Angeles, Jan. 1994 (to be published).

${ }^{10} \mathrm{R}$. Engelmann, C. Shieh, and C. Shu, in Quantum Well Lasers, edited by P. Zory (Academic, New York, 1993), p. 154.

${ }^{11} \mathrm{~J}$. Ralston, S. Weisser, I. Esquivias, E. Larkins, J. Rosenzweig, P. Tasker, and J. Fleissner, J. Quantum. Electron. 29, 1648 (1993).

${ }^{12}$ T. Chen, B. Zhao, Y. Yamada, Y. Zhuang, and A. Yariv, Electron Lett. 28, 1989 (1992).

${ }^{13}$ R. Nagarajan, T. Fukushima, J. E. Bowers, R. S. Geels, and L. A. Coldren, Electron Lett. 27, 1058 (1991).

${ }^{14}$ K. Y. Lau and N. Bar-Chaim, Photon. Tech. Lett. 4, 118 (1992). 